\title{
Making Conditionally Secure Cryptosystems Unconditionally Abuse-Free in a General Context
}

(Extended Abstract)

\author{
Yvo G. Desmedt \\ Dept. EE E CS, Univ. of Wisconsin - Milwaukee \\ P.O. Box 784, WI 53201 Milwaukee, U.S.A.
}

Abstract. [Sim84] introduced the concept of subliminal channel in the context of signature systems. [Des88b] presented a solution against subliminal channels and extended in [Des88a] the solution to abuse-free coin-flipping, abuse-free generation of public keys, and abuse-free zero-knowledge. In this paper we demonstrate that a whole family of systems (generalized Arthur-Merlin games) can be made abuse-free, avoiding the exhaustive approach of [Des88a]. We will hereto formalize the concept of abuse.

\section{Introduction}

[Sim84] found that a secret message can be hidden in a subliminal way through the authentication process. Simmons called the hidden channel the subliminal channel Simmons illustrated it, by comparing it with two prisoners who are communicating authenticated messages in full view of a warden. The warden is able to read the messages. The subliminal consists in hiding a message through the authentication scheme such that the warden cannot detect its use nor read the hidden part (for other subliminal channels see [JS86,Sim85,Sim86]).

[Des88b] demonstrated that subliminal-free authentication and signature systems can be made by introducing the concept of active warden (a warden who modifies the authenticator). [Des88a] studied subliminal channels in different contexts (which was then called abuses). [Des88a]'s solutions against abuses are exhaustive, discussing particular solutions to particular problems. The goal of this paper is to prove, in a constructive way, that all cryptosystems can be made abuse-free using a compiler which will transform a given cryptosystem into an abuse-free version (similarly as [GMW86, p. 185], but keeping the solution practical). However, our solution could ruin the security specifications, in other words, it could be that the abuse-free version is no longer a secure cryptosystem. Therefore, we can only prove our theorem for a family of cryptosystems. 


\section{Formal model for abuses and abuse-freeness}

We assume that the reader is familiar with terminology of [GMR89] (will be briefly overviewed in final paper) and notations in [CEvdGP87]. We now introduce a formal description of an active and a passive warden, covering them together to avoid longwindedness. The model has similarities with the one in [BOGKW88].

Definition 1 Let $A_{1}, \ldots, A_{m}, A_{m+1}$ be probabilistic Turing machines. If all machines $A_{i}$ have a shared common read-only tape $C$ and in addition:

- all $A_{i}(1 \leq i \leq m+1)$ have their own work-tape and own random tape,

- each $A_{i}(1 \leq i \leq m+1)$ has a private read-only tape $H_{i}$ and the content of these tapes has ben written before the machines run,

- for all $i$ such that $1 \leq i \leq m, A_{i}$ has $m-1$ write-only communication tapes $T_{i, j}$ $(1 \leq j \leq m$ and $i \neq j)$ and $A_{m+1}$ has $m(m-1)$ read only communication tapes $R_{i, j}^{\prime}$ such that $R_{i, j}^{\prime}=T_{i, j}$ (for all $i$ and $j$ such that $i \neq j$ and $1 \leq i \leq m$ and $1 \leq j \leq m)$

- for all $i$ such that $1 \leq i \leq m, A_{i}$ has $m-1$ read-only communication tapes $R_{i, j}$ $(1 \leq j \leq m$ and $i \neq j)$ and $A_{m+1}$ has $m(m-1)$ write only-communication tapes $T_{i, j}^{\prime}$ such that $T_{i, j}^{\prime}=R_{i, j}$ (for all $i$ and $j$ such that $i \neq j$ and $1 \leq i \leq m$ and $1 \leq j \leq m)$,

- the order in which these machines write on the tapes is fixed (e.g., $A_{1}$ starts),

then we call $\mathbf{A}=\left(A_{1}, \ldots, A_{m}, A_{m+1}\right)$ an $m$-participant system with warden, $A_{m+1}$, or for short m-participant system. We will denote $A_{m+1}$ mostly as $W$. If $m$ could be an indeterminate, we call $\mathbf{A}$ a multi-participant system with warden, $W$. If the warden, $W$, does not read the common tape $C$, his random tape, his private read-only tape $H_{m+1}$; but only writes on tape $T_{i, j}^{\prime}$ the same symbol which he reads from tape $R_{j, i}^{\prime}$ (for all $i$ and $j, i \neq j$ ), then we say that the warden is passive. In all other cases we say that the warden, $W$, is active.

We remark that the case that the actual communication links don't exist corresponds with machines that don't write on these tapes. Adapting the above model could be useful in other contexts. The private read-only tape $H_{i}$ can be used e.g., to store secret keys. We assume mostly that the length of $H_{i}$ is polynomial in $x$, where $x$ is the common input. Simplification of notations is possible by giving each $A_{i}(1 \leq i \leq m)$ only one tape $T_{i}$ and one tape $R_{i}$. However, in our model in the case that the warden is passive the network corresponds with a complete directed graph. It depends on the context which power the probabilistic Turing machines $A_{1}, \ldots, A_{m}$ and $W$ have. 
Definition 2 Let $S(x, \mathbf{A})$ be a predicate, (specifications of the security), where $\mathbf{A}$ is a $k$-participant system and $x \in\{0,1\}^{*}$. If for a multi-participant system, $\mathbf{B}$ holds that $S(x, \mathbf{B})=1$ for all sufficiently long (large) $x \in L$, where $L \subset\{0,1\}^{*}$, then we call B an $S(L)$-system with (active or passive) warden, $W$, or shorter an $S$-system if there is no ambiguity. We will also say that the $m$-participant system $\mathbf{B}$ is $S$-secure.

Definition 2 allows us to speak about authentication-systems, signature-systems and so on. For our purposes, we now adapt notations introduced in [GMR89]. For a run of $\mathbf{A}$, an $m$-participant system, with $x$ on the shared common tape $(C)$ and with $h_{l}$ on $A_{l}$ 's private read-only tape, the l-participant's view corresponds with all that can be seen from his random tape and from the read-only communication tapes $R_{l, j}$ (for all $j \in\{1, \ldots, l-1, l+1, \ldots, m+1\}$ ). Let $\operatorname{Pview}_{\mathbf{A}, l}\left(x, h_{l}\right)$ be the random variable whose value is the l-participant's view. If the warden, $W$, is an active one, we define the warden's view to be everything that can be seen from $W$ 's random tape and from all read only communication tapes $R_{i, j}^{\prime}$, for $i \neq j$. If the warden is passive, and $x$ is on the shared common tape $(C)$, we define the warden's view to be everything that can be seen from all read only communication tapes, $R_{i, j}^{\prime}(i \neq j)$. Wview $A(x)$ is the random variable whose value is the warden's view. To simplify notations, we did not specify the input $h_{m+1}$ in the expression Wview $\mathbf{A}(x)$.

Definition 3 Let $\mathbf{A}=\left(A_{1}, \ldots, A_{m}, W\right)$ be an $S(L)$-system, with warden $W$. Let $\mathbf{A}^{\prime}=\left(A_{1}^{\prime}, \ldots, A_{m}^{\prime}, W\right)$ be an $m$-participant system. $\mathbf{A}^{\prime}$ is a perfect (statistical) (computational) abuse of the $S(L)$-system $\mathbf{A}$ if:

1. Warden-indistinguishable: $\left\{\right.$ Wview $\left._{\mathbf{A}}(x)\right\}$ and $\left\{\right.$ Wview $\left._{\mathbf{A}^{\prime}}(x)\right\}$ (families of random variables) are equal (statistically indistinguishable) (computational indistinguishable) on $L^{\prime}$, where $L^{\prime}=L$ if the warden is passive, else $L^{\prime}=\left\{\left(x, h_{m+1}\right)\right\}$ $x \in L$ and $\left.\left|h_{m+1}\right|=|x|^{c}\right\}$.

2. $k$-Participant-distinguishable: $\exists k, h_{k}(1 \leq k \leq m)$ : the families of random variables $\left\{\right.$ Pview $\left._{\mathbf{A}_{\mathbf{k}}^{\prime \prime}, k}\left(x, h_{k}\right)\right\}$ and $\left\{\right.$ Pview $\left._{\mathbf{A}^{\prime}, k}\left(x, h_{k}\right)\right\}$ are not computationally indistinguishable on $L^{\prime \prime}$, where $\mathbf{A}_{\mathbf{k}}^{\prime \prime}=\left(A_{1}^{\prime \prime}, \ldots, A_{\mathrm{m}}^{\prime \prime}, W\right)$ is an $m$-participant system such that $A_{i}^{\prime \prime}=A_{i}$ for all $i \neq k$ and $A_{k}^{\prime \prime}=A_{k}^{\prime}$ and $L^{\prime \prime}=\left\{\left(x, h_{k}\right) \mid x \in\right.$ $L$ and $\left.\left|h_{k}\right|=|x|^{c}\right\}$.

We denote $\mathbf{A}_{\mathrm{k}}^{\prime \prime}$ as: $\mathbf{A} \star A_{k}^{\prime}$ ( $\star$ is non-commutative). The aforementioned participant $k$ is called the subliminal receiver. If there exists an $m$-participant system $\mathbf{A}^{\prime}$, which is an abuse of the $m$-participant system $\mathbf{A}$, we say that $\mathbf{A}$ can be abused.

Definition 4 Let $\mathbf{A}=\left(A_{1}, \ldots, A_{m}, W\right)$ be an $S(L)$-system, with warden $W$. We call $\mathrm{A}$ an abuse-free $S(L)$-system if for all $m$-participant systems $\mathbf{A}^{\prime}=\left(A_{1}^{\prime}, \ldots, A_{m}^{\prime}, W\right)$, $\mathbf{A}^{\prime}$ is not an abuse of the $S(L)$-system $\mathbf{A}$. We call $\mathbf{A}$ a strong (weak) abuse-free $S(L)$ system if for all $m$-participant systems $\mathbf{A}^{\prime}=\left(A_{1}^{\prime}, \ldots, A_{m}^{\prime}, W\right)$ holds $\forall k, h_{k}(1 \leq k \leq$

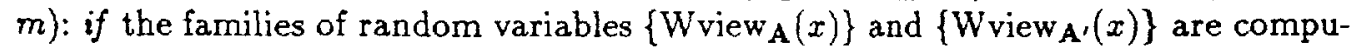
tationally indistinguishable on $L^{\prime}$, then $\left\{\operatorname{Pview}_{\mathbf{A} \star A_{k}^{\prime}, k}\left(x, h_{k}\right)\right\}$ and $\left\{\operatorname{Pview}_{\mathbf{A}^{\prime}, k}\left(x, h_{k}\right)\right\}$ 
are statistically (computationally) indistinguishable on $L^{\prime \prime}$, where $L^{\prime}$ and $L^{\prime \prime}$ were defined in Definition 3.

\section{Informal interpretation}

In an abuse, the passive warden, $W$, is listening to all communications going on during the run of the system. However, the passive warden, $W$, has no access to the tapes $H_{i}$. The subliminal receiver $k$ is waiting to receive hidden information and is therefore running his special program $A_{k}^{\prime}$ instead of running the normal one, namely $A_{k}$.

Abuse-freeness means that one does not exclude that a different system is used than the one intended, but the warden will detect it (almost always). Strong abuse-freeness means that a polynomial-bounded warden will (almost always) detect an abuse even if the other participants have all infinite computer power.

\section{A general solution}

\subsection{A BUILDING BLOCK}

Let us first discuss a slightly modified version of [Des88a] abuse-free generation of public keys (the main difference is that $W$ will always publish the public key). In the final paper we will formally define what a public-key generation system is also considering [GMR88, pp. 290-291]'s definition.

Lemma 1 If $G(\cdot)$ forms a group and $r$ is chosen out of $G$ according to a uniform probability distribution, (the probability to select a given $r$ is $p(r)=1 /|G|$ ), then: $\forall x \in G: p(x \cdot r)=1 /|G|$ and $p(r \cdot x)=1 /|G|$, or $x \cdot r$ and $r \cdot x$, with $x$ fixed, have uniform distributions.

Proof. Trivial: based on group theory and [Sha49].

Theorem 1 If a polynomial-time operation $\oplus$ is defined on $G$ such that $G(\oplus)$ forms a group and $f$ is hard to invert, then the protocol of Figure 1 is a strong abuse-free publickey generation system. The abuse-freeness is unconditional. (If the zero-knowledge protocol used is non-intemctive, the length of the input $|x|$ has an upperbound similar as in (BFM88].)

Proof. (Sketch) First observe that such a zero-knowledge protocol exists, because what has to be proven is an NP problem [GMW86]. (The fact that the zero-knowledge aspect in [GMW86] is based on unproven assumptions does not influence our proof, because its soundness is unconditional.)

Let us call the public-key generation system, which is presented in Figure 1, $\mathbf{A}=(A$, $\left.B_{2}, \ldots, B_{m}, W\right)$. It is sufficient to prove that for all 2-participant systems $\mathbf{A}^{\prime}$ holds that: if $\left\{\right.$ Wview $\left._{\mathbf{A}}(x)\right\}$ and $\left\{\right.$ Wview $\left._{\mathbf{A}^{\prime}}(x)\right\}$ are computationally indistinguishable on $L^{\prime}$ then $\left\{\mathrm{Pview}_{\mathbf{A} \star A_{2}^{\prime}, 2}\left(x, h_{2}\right)\right\}$ and $\left\{\mathrm{Pview}_{\mathbf{A}^{\prime}, 2}\left(x, h_{2}\right)\right\}$ are statistically indistinguishable 
$r \epsilon_{(R)} G$ and $k \epsilon_{(R)} K$ and

$m:=c(r, k)$

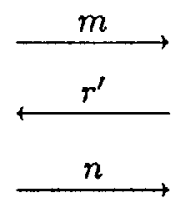

$A$ proves to $W$ that

$\exists r \in G, k \in K$ :

$m=c(r, k) \wedge n=f\left(r \oplus r^{\prime}\right)$,

using zero-knowledge.

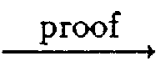

If proof is interactive $W$ asks
questions and proof is
repeated.

$W$ verifies $A$ 's proof. $W$ publishes $A$ 's public key $n$.

FIGURE 1. Abuse-free generation of public key

on $L^{\prime \prime}$, where $x$ specifies a description of $G$ (as its size and so on), and where $L^{\prime}$ and $L^{\prime \prime}$ are similar as in Definition 3. Because the warden doesn't use his private read-only tape $H_{m+1}$, we replace $L^{\prime}$ by $L$ without problems. We denote $\mathbf{A}$ as $\mathbf{A}=(A, B, W)$. Observe that $\left\{\mathrm{Pview}_{\mathrm{A} \times \mathrm{A}_{2}^{\prime}, 2}\left(x, h_{2}\right)\right\}=\left\{\mathrm{Pview}_{\mathbf{A}, 2}\left(x, h_{2}\right)\right\}$ because $B$ is not sending and thus not influencing. We will prove more than required; which is that there exists a poly-size family of circuits $C$ such that if $\left\{\right.$ Wview $\left._{A}(x)\right\}$ and $\left\{W_{\text {view }}(x)\right\}$ are $C$-computationally indistinguishable then, $\left\{\operatorname{Pview}_{\mathbf{A}, 2}\left(x, h_{2}\right)\right\}$ and $\left\{\operatorname{Pview}_{\mathbf{A}^{\prime}, 2}\left(x, h_{2}\right)\right\}$ are statistically indistinguishable on $L^{\prime \prime}$.

Consider the circuit which the warden will use to check the zero-knowledge proof. Let the circuit return a 1 if the warden accepts the proof and a 0 otherwise. For this particular circuit, saying that $\left\{\right.$ Wview $\left._{\mathbf{A}}(x)\right\}$ and $\left\{\right.$ Wview $\left._{\mathbf{A}^{\prime}}(x)\right\}$ are $C$-computational indistinguishable means that for all constants $e>0$ and all sufficiently long strings $x \in L: \mid p$ (warden rejects $)-p^{\prime}$ (warden rejects) $\left.|<| x\right|^{-e}$, where $p^{\prime}$ (warden rejects) and $p^{\prime}$ (warden rejects) denote respectively the probability that the warden will reject (the proof) when $A$ and $A^{\prime}$ is executed. (A correct proof is not necessarily accepted with probability one, due to the definition of completeness, which is important for noninteractive proofs.)

Pview $_{\mathbf{A}^{\prime}, 2}\left(x, h_{2}\right)$ is nothing else than $n$ sent by $W$ and the probability that a specific $n_{i}$ is sent is denoted as $p^{\prime}\left(n=n_{i}\right)$. Similarly $p\left(n=n_{i}\right)$ corresponds to the probability that $B$ receives $n_{i}$ when $A$ is executed.

Using our reformulations, it is sufficient to prove that if (1) holds for all $e>0$ and all sufficiently long $x \in L$, then (2) holds for all $d>0$ and all sufficiently long $x \in L$.

$$
\begin{aligned}
p^{\prime}(\text { warden rejects }) & <|x|^{-e} \\
\sum_{n_{i}}\left|p\left(n=n_{i}\right)-p^{\prime}\left(n=n_{i}\right)\right| & <|x|^{-d}
\end{aligned}
$$


We first describe how $n$ is made. First $A^{\prime}$ makes a string $m$, not necessarily as specified. So there is a probability that $A^{\prime}$ returns a specific $m \in\{0,1\}^{*}$. Then $W$ gives a $r^{\prime} \in G$. Given this $r^{\prime}$ and his previous information, $A^{\prime}$ will make an $n$. $n$ does not necessarily correspond with $f(s)$. Similarly, $A^{\prime}$ will make a proof, but nothing guarantees that this proof is correct. We will denote the string ( $A^{\prime \prime}$ s random, $r^{\prime}, \sigma$ ) as $\alpha$, where $\sigma$ is the string of $W$ 's questions in the zero-knowledge protocol when it is interactive, and when the zero-knowledge proof system is non-interactive, $\sigma$ corresponds with the shared common random string [BFM88]. The string(s) that $A^{\prime}$ sends during the zero-knowledge proof is(are) denoted as $\gamma$. So:

$$
p^{\prime}\left(n=n_{i}\right)=\sum_{m_{j}} \sum_{n_{1}} \sum_{\alpha_{z}} p^{\prime}\left(n=n_{i}, m=m_{j}, \gamma=\gamma_{l} \mid \alpha=\alpha_{z}\right) \cdot p\left(\alpha=\alpha_{z}\right) .
$$

Remark that $p\left(\alpha=\alpha_{z}\right)$ remains the same independently if $A$ or $A^{\prime}$ is executed (and independent of $\left.h_{2}\right)$.

Let us denote $p^{\prime}(\lambda=1)$ the probability that machine $A^{\prime}$ will return at one or another stage of the protocol something different than it should have returned when it would have followed the protocol. We then prove that:

$$
\sum_{n_{i}}\left|p\left(n=n_{i}\right)-p^{\prime}\left(n=n_{i}\right)\right| \leq 2 p^{\prime}(\lambda=1) .
$$

Let us denote $p^{\prime}$ (warden rejects) as $p^{\prime}(\rho=1)$. The problem remaining now is to relate $p^{\prime}(\lambda=1)$ with $p^{\prime}(\rho=1)$ and then to finally prove the theorem.

We prove that:

$$
p^{\prime}(\lambda=1)=1+\frac{p^{\prime}(\rho=1)+p^{\prime}(\rho=0 \mid \lambda=1)-1}{\left(p^{\prime}(\rho=0 \mid \lambda=0)-p^{\prime}(\rho=0 \mid \lambda=1)\right)}
$$

Assuming (1), this means $p^{\prime}(\rho=1)<|x|^{-e}$ for all $e>0$ and $x$ large enough, and using the definition of completeness and soundness we obtain that for all $t>0$ and sufficiently large $x$ : $p^{\prime}(\lambda=1)<|x|^{-t}$. Then follows that if (1) holds for all $e>0$ and all sufficiently long $x \in L$, then (2) holds for all $d>0$ and all sufficiently long $x \in L$.

We have used the symbol $\oplus$ for a visualization aid in case $G=G F\left(2^{n}\right)$, but the group $G$ does not have to be Abelian.

\subsection{OUR SOLUTION}

Let us first introduce a special case of a sequential multi-participant system. (What we describe can be run in parallel under some circumstances, but our definition of a multi-participant system requires an order in which the machines write on their communication tapes.)

Definition 5 Let $\mathbf{A}$ be a sequential $m$-participant system with passive warden $W$. Let us call $x$ the input of the common input tape, $h_{i}$ and $q_{i, j}^{*}$ the content of respectively 
the private tape $H_{i}$ and the read-only communication tape $R_{i, j}$ at stage $s$. The binary string $r_{i}$ contains the string read by $A_{i}$ during the protocol from the random tape. We assume that the length of $h_{i}, q_{i, j}^{s}$ and $r_{i}$ and the number of stages (this last requirement could be relaxed) are polynomial in function of the length of $x . q_{i, j}^{s}$ could be empty. During stage $s, A_{\pi(s)}$ writes $n_{s}$ on tape $T_{\pi(s), \phi(s)}$. If $\pi(s) \neq l(l$ fixed $)$, then $n, \in_{R} G_{x, s}$ or $n_{s}=f_{s}\left(x, q_{\pi(s), 1}^{1}, q_{\pi(s), 2}^{1}, \ldots, q_{\pi(s), m}^{1}, \ldots, q_{\pi(s), 1}^{s-1}, q_{\pi(s), 2}^{s-1}, \ldots, q_{\pi(s), m}^{s-1}\right)$, such that:

- the form of $n$, is known beforehand in a deterministic way,

- $\forall x, s: G_{x, s}(+)$ forms a group, such that in polynomial-time (in function of the length of $x$ ) one can: execute the operations + , check if $x \in G_{x, s}$, and select a random element of $G_{x, s}$,

- the functions $f$, are executable in polynomial time.

If $\pi(s)=l$, then $n_{s}=f_{s}\left(x, r_{l}, h_{l}, q_{l, 1}^{l}, q_{l, 2}^{1}, \ldots, q_{l, m}^{1}, \ldots, q_{l, 1}^{s-1}, q_{l, 2}^{s-1}, \ldots, q_{l, m}^{s-1}\right)$, and the predicate $B_{s}\left(h_{l}\right)=1$ is satisfied, such that:

- checking if an input exists such that $f_{s}$ (input) $=$ output is an NP problem,

- the length of $r_{l}, g$, is fixed for a given $x$.

If $\mathbf{A}$ satisfies all the described properties here; we call $\mathbf{A}$ a generalized Arthur-Merlin game, with $A_{l}$ being Merlin [Bab85].

Observe that if $A_{s}$ is not Merlin its output is either truly random or a deterministic function of its inputs, so in the last case the random tape is not used. We would like to prove now that all $S(L)$-systems which are generalized Arthur-Merlin games can be made abuse-free. However, our solution could ruin its (security) specifications; therefore we can only prove a restricted form of it, which is based on a repetitive use of Theorem 1 . We claim that most practical, conditionally secure cryptosystems can be made abuse-free if one allows interaction with the warden. Giving a proof of this claim is impossible due to a lack of an adequate formal description of all possible cryptosystems.

Corollary 1 If $\mathbf{A}$ is a generalized Arthur-Merlin game and an $S(L)$-system, then there exists a multi-participant system $\mathbf{A}^{\prime}$ (with active warden), such that either:

- $\mathbf{A}^{\prime}$ is an unconditionally strong abuse-free $S(L)$-system, or

- $\mathbf{A}^{\prime}$ isn't an $S(L)$-system.

Proof. Our proof will be constructive by describing the multi-participant system $\mathbf{A}^{\prime}$. A generalized Arthur-Merlin game can be considered to be a system which is mainly publishing public keys, random numbers and/or deterministic calculations. In an initial stage, $A_{l}^{\prime}$ sends $W$ a commitment $\left(m_{l}=c\left(r_{l}, k_{l}\right)\right)$ for the bit string $r_{l}$. 
Then the warden sends his random choice of his bit string $r_{l}^{\prime}$ (with length $g$ ). Let us now describe what is executed in $\mathbf{A}^{\prime}$ instead of the execution of stage $s$ in $\mathbf{A}$. We distinguish three different cases. If $\pi(s) \neq l$ and $n$, has the form $f_{s}\left(x, q_{\pi(s), 1}^{1}, q_{\pi(s), 2}^{1}\right.$, $\left.\ldots, q_{\pi(s), m}^{1}, \ldots, q_{\pi(s), 1}^{s-1}, q_{\pi(s), 2}^{s-1}, \ldots, q_{\pi(s), m}^{s-1}\right)$, then $A_{s}^{\prime}$, sends the warden $n_{s}$ and the warden verifies if $n_{s}$ is correct (if it is not, the warden calculates $n_{s}$ himself) and sends $n_{s}$ (writes $q_{\phi(s), \pi(s)}^{s}=n$, on the tape $T_{\phi(s), \pi(s)}^{\prime}$ ). If $\pi(s) \neq l$, and $n_{s}$ had to be chosen randomly from $G_{x, s}$, then the following steps are executed:

1. $A_{\pi(s)}^{\prime}$ chooses $n_{s} \in_{(R)} G_{x, s}$ and sends $W$ a commitment $\left(m_{\pi(s)}=c\left(n_{s}, k_{\pi(s)}\right)\right)$,

2. the warden sends $A_{\pi(s)}^{\prime}: n_{s}^{\prime}, \in_{R} G_{x, s}$,

3. $A_{\pi(s)}^{\prime}$ sends the warden: $n_{s}$ and $k_{\pi(s)}$.

4. the warden verifies the commitment. If correct, then the warden writes $q_{\phi(s), \pi(s)}^{s}=$ $n_{s}+n_{s}^{\prime}$, else sends a random $q_{\phi(s), \pi(s)}^{s} \in_{R} G_{x, s}$.

If $\pi(s)=l$, then $A_{l}^{\prime}$ sends $n_{s}=f_{s}\left(x, r_{l} \oplus r_{l}^{\prime}, h_{l}, q_{l, 1}^{1}, q_{l, 2}^{1}, \ldots, q_{l, m}^{1}, \ldots, q_{l, 1}^{s-1}, q_{l, 2}^{s-1}, \ldots, q_{l, m}^{s-1}\right)$ (where $\oplus$ is the bit-by-bit exclusive-or) and gives a zero-knowledge proof to $W$ that:

$$
\begin{aligned}
& \exists k_{l} \in K, r_{l} \in\{0,1\}^{g}, h_{l}: \quad m_{l}=c\left(r_{l}, k_{l}\right) \quad \wedge \quad B_{s}\left(h_{l}\right)=1 \\
& \quad \wedge \quad n_{s}=f_{s}\left(x, r_{l} \oplus r_{l}^{\prime}, h_{l}, q_{l, 1}^{1}, q_{l, 2}^{1}, \ldots, q_{l, m}^{1}, \ldots, q_{l, 1}^{s-1}, q_{l, 2}^{s-1}, \ldots, q_{l, m}^{s-1}\right) .
\end{aligned}
$$

The warden verifies then $A_{l}^{\prime \prime}$ s proof and writes $q_{\phi(s), l}^{s}=n_{s}$.

Let us now prove that $\mathbf{A}^{\prime}$ is abuse-free. It holds that $\forall \mathbf{A}^{\prime \prime}:\left\{\operatorname{Pview}_{\mathbf{A}^{\prime} \star A_{i}^{\prime \prime}, i}\left(x, h_{i}\right)\right\}$ and $\left\{\mathrm{Pview}_{\mathbf{A}^{\prime}, i}\left(x, h_{i}\right)\right\}$ are equal, when $i \neq l$; due to the independency of the warden's random choices. Also $\forall \mathbf{A}^{\prime \prime}:\left\{\operatorname{Pview}_{\mathbf{A}^{\prime} \star A_{l}^{\prime \prime}, l}\left(x, h_{l}\right)\right\}$ and $\left\{\mathrm{Pview}_{\mathbf{A}^{\prime \prime}, l}\left(x, h_{l}\right)\right\}$ are equal. This implies that the rest of the proof follows easily from the proof of Theorem 1 (because the remark made after (3) is still valid).

Let us discuss some improvements. Sometimes, it is sufficient for the warden to check once and for all (or at the beginning of the protocol) that $h_{l}$ satisfies the appropriate predicates. Instead of $r_{l}$ being modified at the beginning of the protocol, it could be done during the different stages of the protocol. It is then necessary to guarantee independency of randomness when appropriate and to treat earlier altered random as deterministic variables instead of random ones. If a function $f_{s}$ is deterministic, it means $r_{l}$ is not used, then there is no need for interaction if $A_{l}^{\prime \prime}$ 's zero-knowledge proof is non-interactive. 
We make the important observation that all the unconditionally abuse-free cryptosystems discussed in [Des88a,Des88b] are generalized Arthur-Merlin games and therefore special cases of ours and that therefore the proof of their abuse-freeness has not to be given for each separately.

Let us discuss the consequences of Corollary 1 by analyzing which cryptosystems can be made abuse-free. One first has to realize that, so far, most cryptosystems have been defined without taking a (passive) warden into consideration. So first, one has to convert them into a definition in which the warden's role and privileges are defined. Mostly, one considers such a warden as an opponent. In particular the above corollary also implies (after a careful redefinition, as mentioned) that abuse-free interactive proof systems and zero-knowledge systems for NP languages exist, using GoldreichMicali-Wigderson [GMW86] proof for 3-colourability and Corollary 1, however the verifier's soundness collapses to a conditional soundness, instead of an unconditional one. It is possible to make abuse-free zero-knowledge proofs for all languages in NP such that the soundness of the verifier remains unconditional, as was recently demonstrated [BD89]. This solution is however not based on the above compiler. The protocol described in [Des88a] to make zero-knowledge proof systems for NP languages abuse-free is only conditionally abuse-free, while the one here is unconditional.

\section{Conclusions and open problems}

Our approach to abuse-freeness allows one to make all (generalized Arthur-Merlin games) cryptosystems abuse-free. It is an open problem of whether Corollary 1 can be generalized to more general multi-participant systems (excluding the obvious generalizations). If so, a different proof technique will be necessary.

Trying to apply Corollary 1 on unconditionally secure authentication systems as e.g., [GMS74], ruins the unconditionality of the authentication. The question whether it can be solved in one or another way is an open problem, even if the abuse-freeness would only be weak abuse-freeness (as defined). A similar remark was made related to soundness of zero-knowledge schemes, there also the unconitionality of the soundness was ruined by using above compiler. For zero-knowledge, the same problem could be solved [BD89], but the solution is not based on the above compiler.

The approach followed in this paper is constructive as well as general. It avoids the exhaustive character followed in [Des88a] but gives a global solution to the problem, useful for many situations. One of the advantages of this approach is the reduction of proofs required to demonstrate the abuse-freeness of different cryptosystems to mainly one proof. 


\section{REFERENCES}

[Bab85] L. Babai. Trading group theory for randomness. In Proceedings of the seventeenth ACM Symp. Theory of Computing, STOC, pp. 421-429, May 6-8, 1985.

[BD89] M. V. D. Burmester and Y. G. Desmedt, June 1989. Text in preparation.

[BFM88] M. Blum, P. Feldman, and S. Micali. Non-interactive zero-knowledge and its applications. In Proceedings of the twentieth ACM Symp. Theory of Computing, STOC, pp. 103-112, May 2-4, 1988.

[BOGKW88] M. Ben-Or, S. Goldwasser, J. Kilian, and A. Wigderson. Multi-prover interactive proofs: How to remove intractability assumptions. In Proceedings of the twentieth ACM Symp. Theory of Computing, STOC, pp. 113-131, May 2-4, 1988.

[CEvdGP87] D. Chaum, J.-H. Evertse, J. van de Graaf, and R. Peralta. Demonstrating possession of a discrete logarithm without revealing it. In A. Odlyzko, editor, Advances in Cryptology. Proc. Crypto'86 (Lecture Notes in Computer Science 263), pp. 200-212. Springer-Verlag, 1987. Santa Barbara, California, U.S.A., August 11-15.

[Des88a] Y. Desmedt. Abuses in cryptography and how to fight them. Presented at Crypto'88, Santa Barbara, California, U.S.A., to appear in: Advances in Cryptology. Proc. of Crypto'88 (Lecture Notes in Computer Science), Springer-Verlag, August 1988.

[Des88b] Y. Desmedt. Subliminal-free authentication and signature. In C. G. Günther, editor, Advances in Cryptology, Proc. of Eurocrypt'88 (Lecture Notes in Computer Science 330), pp. 23-33. Springer-Verlag, May 1988. Davos, Switzerland.

[GMR88] S. Goldwasser, S. Micali, and R. Rivest. A digital signature scheme secure against adaptive chosen-message attacks. Siam J. Comput., 17(2), pp. 281-308, April 1988.

[GMR89] S. Goldwasser, S. Micali, and C. Rackoff. The knowledge complexity of interactive proof systems. Siam J. Comput., 18(1), pp. 186-208, February 1989.

[GMS74] E. Gilbert, F. MacWilliams, and N. Sloane. Codes which detect deception. The BELL System Technical Journal, 53(3), pp. 405-424, March 1974. 
[GMW86] O. Goldreich, S. Micali, and A. Wigdérson. Proofs that yield nothing but their validity and a methodology of cryptographic protocol design. In The Computer Society of IEEE, 27th Annual Symp. on Foundations of Computer Science (FOCS), pp. 174-187. IEEE Computer Society Press, 1986. Toronto, Ontario, Canada, October 27-29, 1986.

[JS86] T. C. Jones and J. Seberry. Authentication without secrecy. ARS Combinatoria, 21(A), pp. 115-121, May 1986.

[Sha49] C. E. Shannon. Communication theory of secrecy systems. Bell System Techn. Jour., 28, pp. 656-715, October 1949.

[Sim84] G. J. Simmons. The prisoners' problem and the subliminal channel. In D. Chaum, editor, Advances in Cryptology. Proc. of Crypto 83, pp. 51-67. Plenum Press N.Y., 1984. Santa Barbara, California, August 1983.

[Sim85] G. J. Simmons. The subliminal channel and digital signatures. In T. Beth, N. Cot, and I. Ingemarsson, editors, Advances in Cryptology. Proc. of Eurocrypt 8 \& (Lecture Notes in Computer Science 209), pp. 364-378. Springer-Verlag, Berlin, 1985. Paris, France, April 9-11, 1984.

[Sim86] G. J. Simmons. The secure subliminal channel (?). In H. C. Williams, editor, Advances in Cryptology. Proc. of Crypto 85 (Lecture Notes in Computer Science 218), pp. 33-41. Springer-Verlag, 1986. Santa Barbara, California, August 18-22, 1985. 